\title{
Diagnostic Validity of 3D-Computed Tomographic Angiography in Spontaneous
} Subarachnoid Haemorrhage

\author{
Nur Mohammad', Jalal Uddin Mohammad Rumi², Sadia Jabeen Khan ${ }^{3}$, Kalim Uddin ${ }^{4}$, \\ Patoary Mohammed Faruque ${ }^{5}$
}

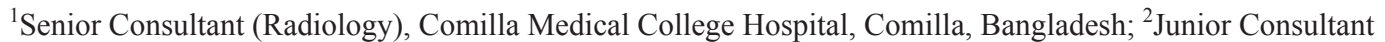
(Neurosurgery), National Institute of Neurosciences and Hospital, Dhaka, Bangladesh; ${ }^{3}$ Junior Consultant (Obstetrics \& Gynaecology), National Institute for Cancer Research \& Hospital, Dhaka, Bangladesh; ${ }^{4}$ Registrar (Neurotrauma), National Institute of Neurosciences and Hospital, Dhaka, Bangladesh; ${ }^{5}$ Consultant Neurosurgeon, Bangladesh Specialized Hospital, Dhaka, Bangladesh
\end{abstract}

[Received: 1 October 2018; Accepted: 12 November 2018; Published: 1 January 2019]

\begin{abstract}
Background: Diagnostic validity of different tests for the detection of spontaneous subarachnoid haemorrhage is an important issue. Objectives: The purpose of the present study was to validate 3D-Computed tomographic angiography in spontaneous subarachnoid haemorrhage. Methodology: This cross-sectional study was carried out in the Department of Neurosurgery and Cath Lab of DMCH in collaboration with private diagnostic centre from September 2013 to February 2015 for a period of six (06) months. Adult patients diagnosed as a case of spontaneous SAH based on clinical features and confirmed by plain CT evidence of subarachnoid blood were included as study population. Patients having current history of trauma, poor clinical grade and agitated patient, patient with renal insufficiency, known allergy to iodinated contrast agent and patients who were not willing participate in the study were excluded from this study. Then both CT angiography and DSA were performed to detect cause of bleeding and to make a treatment planning. In this study DSA was considered as reference standard for evaluation of CTA. Sensitivity, specificity, positive predictive value, negative predictive value and diagnostic accuracy of CTA were calculated per patient basis and per aneurysmal basis. Result: A total number of 37 patients presented with spontaneous subarachnoid haemorrhage were recruited for this study. The mean age of patients was $58.53 \pm 7.54$ years. Sensitivity and specificity in depicting intracranial aneurysms were, $93.75 \%$ and $100 \%$ respectively on a per-patient basis. Positive predictive value and negative predictive value of CTA are $100 \%$ and $71.43 \%$ respectively. CTA had shown $94.59 \%$ accuracy in detection of intracranial aneurysm. Sensitivity and specificity in depicting intracranial aneurysms were $94.74 \%$ and $100 \%$ respectively on a per-aneurysm basis. Positive predictive value and negative predictive value of CTA are $100 \%$ and $71.43 \%$ respectively. CTA had shown $95.35 \%$ accuracy in detection of intracranial aneurysm. Conclusion: In conclusion CTA has high detection capacity of aneurysm among spontaneous subarachnoid haemorrhage patients.[Journal of National Institute of Neurosciences Bangladesh, 2019;5(1): 47-52]
\end{abstract}

Keywords: Diagnostic Validity; 3D-Computed Tomographic Angiography; Spontaneous Subarachnoid Haemorrhage

Correspondence: Dr. Nur Mohammad, Senior Consultant (Radiology), Comilla Medical College Hospital, Comilla, Bangladesh; Email: nurmdr.radiol@gmail.com; Cell no.:+8801711158381

Conflict of interest: No conflict of interest is declared by the authors relevant to this paper which is subjected to disclose.

Funding agency: This research project was not funded by any group or any institution.

Contribution to authors: Mohammad N, Rumi JUM contributed from the protocol preparation, data collection up to report writing. Manuscript writing was performed by Mohammad N \& Rumi JUM. Statistical analysis was performed by Mohammad N, Rumi JUM. Khan SJ, Uddin K, Faruque PM involved in revision of manuscript.

How to cite this article: Mohammad N, Rumi JUM, Khan SJ, Uddin K, Faruque PM. Diagnostic Validity of 3D-Computed Tomographic Angiography in Spontaneous Subarachnoid Haemorrhage. J Natl Inst Neurosci Bangladesh, 2019;5(1): 47-52

Copyright: C2019. Mohammad et al. Published by Journal of National Institute of Neurosciences Bangladesh. This article is published under the Creative Commons CC BY-NC License (https://creativecommons.org/licenses/by-nc/4.0/). This license permits use, distribution and reproduction in any medium, provided the original work is properly cited, and is not used for commercial purposes.

\section{Introduction}

Early diagnosis and detection of the underlying cause are considered critical in order to favour the prognosis among spontaneous subarachnoid haemorrhage (SAH). Early identification of an underlying ruptured aneurysm and adequate treatment may eliminate the risk of 
re-bleeding, which is a major mortality factor ${ }^{1}$. The therapeutic alternatives of a ruptured aneurysm include surgical clipping and endovascular coiling. Intra-arterial digital subtraction angiography (DSA) is considered the imaging gold standard for depicting the presence of an intracranial aneurysm. However, DSA is an invasive imaging modality, with a relatively high cost and carries a small risk of neurological complications. DSA has currently been reported to cause transient or permanent neurological deficits when performed in 1 and $0.5 \%$ of patients, respectively ${ }^{2}$. Therefore, the utility of a non-invasive imaging modality that can detect the presence of intracranial aneurysms is of particular clinical significance.

MRA has been an attractive alternative to conventional angiography due to its risk free, noninvasive nature. Added advantages of this technique are its ability to produce images in three dimensions and the ability to produce many projections after a single acquisition, and the technique forms a routine part of an MRI investigation for cerebral aneurysms ${ }^{3}$. However, a variety of artifacts by phase or magnitude variations in the MR signal afflict MRA and has limited its use, especially in preoperative planning of vascular malformations and aneurysms ${ }^{4}$.

Currently, the advent of CTA has been used in the diagnosis and preoperative planning for patients presenting with aneurysmal $\mathrm{SAH}^{5}$. It has been suggested that CTA has several advantages over MRA. CTA possesses greater resolution than $\mathrm{MRA}^{6}$; CTA is unique in its capacity to display the relationships of arteries and aneurysms to bone, which contributes greatly to preoperative planning for patients presenting with aneurysmal $\mathrm{SAH}^{7}$; CTA can be performed in seconds and can be performed immediately after the unenhanced CT has confirmed an $\mathrm{SAH}^{8}$.

Indeed, with continued improvements in imaging, hardware and concomitant software enhancements, the sensitivity and specificity of CTA approaches that of DSA $^{9}$. However, whether or not CTA can be used as the sole modality to provide sufficient diagnostic information to guide the management of non-traumatic SAH still needs further clinical evidence.

The present study aims to assess the diagnostic efficacy of CTA in a preoperative clinical setting in patients with SAH with emphasis in detection of aneurysms. The role of CTA included the identification of the bleeding source, the detection of additional vascular abnormalities and the ability of planning a pretreatment therapy. The purpose of the present study was to validate 3D-Computed tomographic angiography in spontaneous subarachnoid haemorrhage.

\section{Methodology}

This cross-sectional study was carried out in the Department of Neurosurgery and Cath Lab of Dhaka Medical College Hospital (DMCH), Dhaka, Bangladesh in collaboration with private diagnostic centre from September 2013 to February 2015 for a period of six (06) months. Purposive and convenient sampling technique was used to collect the patients. Adult patients diagnosed as a case of spontaneous SAH based on clinical features and confirmed by plain CT evidence of subarachnoid blood were included as study population. Patients having current history of trauma, poor clinical grade and agitated patient, patient with renal insufficiency, known allergy to iodinated contrast agent and patients who were not willing participate in the study were excluded from this study. In the study period all patients of neurosurgery department of Dhaka Medical College who fulfill the inclusion criteria were included in the study. The study was started in neurosurgery ward. Clinical features of the sample were recorded in data collection sheet. Then both CTA and DSA were done in order to detect the cause of bleeding and make a treatment planning. All spiral CTAs were performed on a helical CT Scanner. One-hundred milliliters of non-ionic iodinated contrast medium was given by peripheral electric pump injector at a rate of 4 $\mathrm{ml} / \mathrm{s}$. The scanning area was from the level of $\mathrm{C} 1$ up to the cranial vault. Therefore, posterior inferior cerebellar arteries (PICAs) was always included in the study regardless their origin. After data acquisition and raw data analysis, further 2D [multi-planar reformation (MPR)], and 3D volume rendered reconstruction was done. The CTA film was interpreted. Four vessels DSA were performed via a femoral approach in DMCH Cath Lab. All DSAs was performed and interpreted by the interventional neurosurgical team. The team was blind about the CTA finding. Ruptured aneurysms were treated either surgically or by endovascular coiling. CTA results were compared with DSA findings in all cases. Data were analyzed using standard contingency tables. Patients with negative CTA and DSA results were served as true negatives. Patients with positive CTA and DSA results were served as true positives. CTA false negatives were defined as negative CTA results in cases with vessel pathology found by using DSA. If CTA is positive for an aneurysm but DSA is negative, DSA was further evaluated before declaring CTA as false positive. Accuracy, sensitivity, specificity, positive predictive value and negative predictive value 
of CTA in the detection of intracranial aneurysms were evaluated using DSA as reference standard. Appropriate data were collected by using a preformed data sheet (annexure-1). All other needed data were collected from history sheet and investigation papers. Data were modified to be compatible to input in SPSS program version- 21. Statistical analysis was performed by using a commercially available statistical package (SPSS version 21; SPSS, Chicago, Ill). Quantitative variables were expressed as mean \pm standard deviation, and categorical variables were expressed as frequencies or percentages. When a $\mathrm{p}$ value is $<0.05$, the difference was considered statistically significant. In this study, DSA was considered a diagnostic standard for the evaluation of cerebral aneurysms. Sensitivity, specificity, and accuracy of CT angiography in depicting aneurysms were calculated on per- aneurysm and per-patient basis. Approval from the Institutional review board of $\mathrm{DMCH}$ was taken before commencement of this study.

\section{Results}

A total number of 37 patients presented with spontaneous subarachnoid haemorrhage were recruited for this study after fulfilling the inclusion and exclusion criteria.

Table 1: Age distribution of the study population

\begin{tabular}{lcc}
\hline Age Group & Frequency & Percent \\
\hline$<60$ Years & 18 & 48.6 \\
$>61$ Years & 19 & 51.4 \\
Total & $\mathbf{3 7}$ & $\mathbf{1 0 0 . 0}$ \\
Mean \pm SD (Range) & $58.53 \pm 7.54(41-72$ years)
\end{tabular}

The mean age of patients was $58.53 \pm 7.54$ years; majority age group was 60 to 72 years which was $51.35 \%$. Minimum age was 41 years and maximum age was 72 years (Table 1).

Table 2: Results of CT Angiography in Depicting Aneurysms as per patient basis

\begin{tabular}{lccc}
\hline CTA & \multicolumn{2}{c}{ DSA Finding } & Total \\
\cline { 2 - 3 } Finding & Positive & Negative & \\
\hline Positive & $30(93.8 \%)$ & $0(0.0 \%)$ & $30(81.1 \%)$ \\
Negative & $2(6.2 \%)$ & $5(100.0 \%)$ & $7(18.9 \%)$ \\
Total & $\mathbf{3 2}(\mathbf{1 0 0 . 0 \% )}$ & $\mathbf{5 ( 1 0 0 . 0 \% )}$ & $\mathbf{3 7 ( 1 0 0 . 0 \% )}$ \\
\hline
\end{tabular}

CT angiography and DSA were both positive in aneurysms in 30 patients on a per-patient basis. CTA and DSA were both negative in 5 cases. However, CTA negative but DSA positive were in 2 cases (Table 2).

Table 3: Results of CT Angiography in Depicting Aneurysms as per aneurysm basis

\begin{tabular}{lccc}
\hline CTA & \multicolumn{2}{c}{ DSA finding } & Total \\
\cline { 2 - 3 } Finding & Positive & Negative & \\
\hline Positive & $36(94.7 \%)$ & $0(0.0 \%)$ & $36(83.7 \%)$ \\
Negative & $2(5.3 \%)$ & $5(100.0 \%)$ & $7(16.3 \%)$ \\
Total & $\mathbf{3 8}(\mathbf{1 0 0 . 0} \%)$ & $\mathbf{5 ( 1 0 0 . 0 \% )}$ & $\mathbf{4 3 ( 1 0 0 . 0 \% )}$ \\
\hline
\end{tabular}

CT angiography and DSA were both positive in aneurysms in 36 intracranial aneurysms on a per-aneurysm basis. CTA and DSA were both negative in 5 cases. However, CTA negative but DSA positive were in 2 cases (Table 3 ).

Table 4: Diagnostic Validity of CTA for Detection of aneurysms

\begin{tabular}{lcc}
\hline Diagnostic Validity & Patient Basis & Aneurysm Basis \\
\hline Sensitivity & $93.75 \%$ & $94.74 \%$ \\
Specificity & $100.0 \%$ & $100.0 \%$ \\
PPV & $100.0 \%$ & $100.0 \%$ \\
NPV & $71.43 \%$ & $71.43 \%$ \\
Accuracy & $94.59 \%$ & $95.35 \%$ \\
\hline
\end{tabular}

$\mathrm{PPV}=$ Positive predictive value; $\mathrm{NPV}=$ negative predictive value

Sensitivity and specificity in depicting intracranial aneurysms were, $93.75 \%$ and $100 \%$ respectively on a per-patient basis. Positive predictive value and negative predictive value of CTA are $100.0 \%$ and $71.43 \%$ respectively. CTA had shown $94.59 \%$ accuracy in detection of intracranial aneurysm. Sensitivity and specificity in depicting intracranial aneurysms were, respectively $94.74 \%$ and $100 \%$ on a per-aneurysm basis. Positive predictive value and negative predictive value of CTA are $100 \%$ and $71.43 \%$ respectively. CTA had shown $95.35 \%$ accuracy in detection of intracranial aneurysm (Table 4).

\section{Discussion}

CTA and DSA are ionizing radiation imaging techniques indispensable for the management of patients with $\mathrm{SAH}$, albeit inducing a radiation risk to the patient ${ }^{5}$. The expected additional benefit in diagnostic performance, with the use of CTA, justifies the additional radiation risk. Performance characteristics of diagnostic methods involve in our case the presence or absence of an aneurysm. While usually DSA is considered the gold standard that other imaging methods ought to be compared against, it exhibited a number of false-negative results when 
surgical confirmation was employed ${ }^{7}$. While DSA and CTA combined can provide us with detailed information on the morphological features of the aneurysm, their accuracy can be measured in an undisputed manner only when surgical findings are available.

The present study aims to assess the diagnostic efficacy of CTA in a preoperative clinical setting in patients with SAH with emphasis in detection of aneurysms. In the study period all patients of neurosurgery department of Dhaka Medical College Hospital who fulfill the inclusion and exclusion criteria was included in the study. Then both CTA and DSA were done in order to detect the cause of bleeding and make a treatment planning.

This study group initially included 40 patients with spontaneous SAH. Three patients passed away immediately after CTA scan, thus excluded from the study. Thus there were a total of 37 patients that underwent both CTA and DSA and formed our study group.

The mean age of patients was 58.53( \pm 7.54$)$ years; in a range of 41 to 72 years. In study of Karamessini et al ${ }^{10}$ mean age was $49 \pm 15$ years with age range of 15 to 76 years.

All patients subsequently underwent DSA. All the positive finding revealed in CTA was also detected in DSA. In addition, DSA identified 2 aneurysms in 2 patients who are negative for any abnormality in CTA. Thus out of 37 patients DSA revealed Aneurysm in $32(86.49 \%)$ patients, AVM in 02(05.41\%) patients and negative findings in $03(8.11 \%)$ patients. Out of 32 patients in whom Aneurysm was detected by DSA, $27(85 \%)$ patients had single Aneurysm, in 04(12\%) patients double Aneurysm found and in 1(3\%) patient there were 3 Aneurysm. Thus 38 aneurysms were identified in DSA. In a similar study by Chen et $\mathrm{al}^{11}$ a total of 92 aneurysms were identified in which 80 patients (93\%) harbored one aneurysm and 6 patients (7\%) had 2 aneurysms. One of the newly diagnosed aneurysm was in posterior circulation and size was 7 $\mathrm{mm}$ and the other one was an ICA aneurysm measuring $4 \mathrm{~mm}$ from neck to dome. Among the 38 Aneurysm, $11(28.95 \%)$ were between $2-5 \mathrm{~mm}$ in size, $16(42.11 \%)$ were between $6-9 \mathrm{~mm}$ in size and $11(28.95 \%)$ were $\geq$ $10 \mathrm{~mm}$ in size. Chen $\mathrm{W}$ et $\mathrm{al}^{11}$ showed CTA for detecting aneurysms $<4 \mathrm{~mm}$, between 4 and $10 \mathrm{~mm}$, and $>10 \mathrm{~mm}$ was $96.0 \%, 98.1 \%$ and $100.0 \%$, respectively, on a per-aneurysm basis. Mean of the size of the aneurysms is $6.75 \mathrm{~mm}$ with $2.94 \mathrm{~mm}$ standard deviation in a range of 2 to $12 \mathrm{~mm}$.
In this study CTA correctly detected 36/38 aneurysms. CT angiography depicted positive aneurysms in 30 patients on a per-patient basis and 36 intracranial aneurysms on a per-aneurysm basis. Sensitivity and specificity in depicting intracranial aneurysms were, respectively, $93.75 \%$ and $100.0 \%$ on a per-patient basis and $94.74 \%$ and $100 \%$ on a per-aneurysm basis. CTA showed very high sensitivity (97.9\%), and its specificity was $100.0 \%$. There were 2 aneurysms, which were not detected by CTA. Thus accuracy was $94.59 \%$, with positive and negative predictive values were $100.0 \%$ and $71.43 \%$ respectively.

Chen et $\mathrm{al}^{11}$ study showed the sensitivity, specificity, and accuracy of 16-slice CTA for detecting aneurysms were $97.8 \%, 100.0 \%$ and $98.7 \%$, respectively, on a per-aneurysm basis. Karamessini et a ${ }^{10}$ study showed the sensitivity of CTA for the detection of all aneurysms versus surgery was $88.7 \%$, the specificity $100.0 \%$, the positive predictive value (PPV) $100 \%$, the negative predictive value (NPV) $80.7 \%$ and the accuracy $92.3 \%$. In study of Taschner et $\mathrm{al}^{12}$ showed sensitivity and specificity for CTA were $100 \%$ and $83 \%$, respectively, on a per-aneurysm-basis.

With regard to the location of aneurysms, CTA had a sensitivity of $100 \%$ in the detection of aneurysms in the anterior communicating artery and in the middle cerebral artery bifurcation, regardless their size. This observation is in line with the studies of Anderson et $\mathrm{al}^{13}$ and Velthuis et $\mathrm{al}^{14}$, who also suggested that it is safe to operate based on CTA findings alone, in patients having aneurysms in the aforementioned areas. The results indicate a relative poor detection rate in the posterior circulation aneurysms; this limitation of CTA is well recognized by previous studies ${ }^{14}$. That was mainly due to their small size $(<4 \mathrm{~mm})$, lobulated shape and attachment to the posterior clinoid process, having similar density values with the bones, which made their discrimination difficult. We also observed that the ability to demonstrate an aneurysm in the cavernous or terminal segment of the internal carotid artery and to differentiate the sac from the surrounding bony structures, blood clots and fibrous tissue, demands a high quality examination and careful analysis of the three-dimensional and axial-source images. There was also a difficulty in patients with multiple aneurysms and in distal-branch aneurysms sited out of the scanning field of view. All these factors influenced sensitivity in a negative manner. Even though the sensitivity values for the detection of intracranial aneurysms regardless their size, favor CTA versus DSA, the apparent difference between them is 
not statistically significant $(\mathrm{P}>0.05)$.

The findings of this study was concordant with previous reports ${ }^{15-16}$. Donmez et $\mathrm{al}^{16}$ found that the overall sensitivity, specificity, and accuracy of CT angiography on a per-aneurysm basis were $95.1 \%$, $94.1 \%$, and $95.0 \%$, respectively. In that same series, for aneurysms of less than $3 \mathrm{~mm}$, the sensitivity, specificity, and diagnostic accuracy were $86.1 \%$, $94.1 \%$, and $88.6 \%$, respectively. Tipper et $\mathrm{al}^{17}$ reported the sensitivity and specificity of 16-channel CT angiography as $96 \%$ and $100 \%$, respectively; 51 of 53 aneurysms were detected. Another study with 16-channel multi-detector $\mathrm{CT}$ has been reported by Yoon et $\mathrm{al}^{18}$. They examined 71 patients with subarachnoid hemorrhage, and the overall sensitivity, specificity, and accuracy of CT angiography on a per-aneurysm basis were $92.5 \%, 93.3 \%$, and $92.6 \%$, respectively, for both independent readers. For aneurysms of less than $3 \mathrm{~mm}$, CT angiography had a sensitivity of $74.1 \%$ for reader 1 and of $77.8 \%$ for reader 2 . White et $\mathrm{al}^{19}$ demonstrated a per-patient sensitivity of $92 \%$ and a per-patient specificity of $94 \%$ for the detection of aneurysms, compared with selective cerebral angiography. The per-aneurysm sensitivity was greater for depicting aneurysms larger than $3 \mathrm{~mm}$ than for depicting aneurysms $3 \mathrm{~mm}$ or smaller $(96 \%$ vs 61\%). A recently published meta-analysis ${ }^{20}$ on the detection of intracranial aneurysms with CT angiography also supports our current findings.

Regarding the diagnostic strategy in assessing patients with possible ruptured intracranial aneurysms, the first step is the early clinical assessment and confirmation of SAH with plain CT or lumbar puncture. Immediately afterwards, in the absence of contraindications to contrast material, cerebral CTA should be performed as an easy to carry out method that provides a reliable early diagnosis when positive. When CTA finding is negative but an aneurysm is likely to be present, DSA should be done.

\section{Conclusion}

In conclusion the sensitivity and specificity of CTA is very high for the detection of aneurysm among the spontaneous subarachnoid haemorrhage patients. Accuracy of CTA is also reported with a very high value. During comparing the CTA in patients and aneurysm basis it has been found that in both cases CTA is very high for the detection of aneurysm. A large scale study should be carried out to avoid the selection bias.

\section{References}

1. Ross N, Hutchinson PJ, Seeley H, Kirkpatrick PJ. Timing of surgery for supratentorial aneurysmal subarachnoid haemorrhage: report of a prospective study. Journal of Neurology, Neurosurgery \& Psychiatry. 2002;72(4):480-4.

2. Willinsky RA, Taylor SM, terBrugge K, Farb RI, Tomlinson G, Montanera W. Neurologic complications of cerebral angiography: prospective analysis of 2,899 procedures and review of the literature. Radiology. 2003;227(2):522-8.

3. Jäger HR, Mansmann U, Hausmann O, Partzsch U, Moseley IF, Taylor WJ. MRA versus digital subtraction angiography in acute subarachnoid haemorrhage: a blinded multireader study of prospectively recruited patients. Neuroradiology. 2000; 42(5): 313-26.

4. Yucel EK. Pulmonary MR angiography: is it ready now? Radiology. 1999;210(2):301-3

5. Dorsch NW, Young N, Kingston RJ, Compton JS. Early experience with spiral $\mathrm{CT}$ in the diagnosis of intracranial aneurysms. Neurosurgery. 1995;36(1):230-8

6. Schievink WI, Riedinger M, Maya MM. Frequency of incidental intracranial aneurysms in neurofibromatosis type 1. American Journal of Medical Genetics Part A. 2005;134(1):45-8

7. Truwit CL. CT angiography versus MR angiography in the evaluation of acute neurovascular disease. Radiology. 2007;245(2):362-6.

8. Adams WM, Laitt RD, Jackson A. The role of MR angiography in the pretreatment assessment of intracranial aneurysms: a comparative study. American Journal of Neuroradiology. 2000;21(9):1618-28.

9. Chappell ET, Moure FC, Good MC. Comparison of computed tomographic angiography with digital subtraction angiography in the diagnosis of cerebral aneurysms: a meta-analysis. Neurosurgery. 2003;52(3):624-31.

10. Karamessini MT, Kagadis GC, Petsas T, Karnabatidis D, Konstantinou D, Sakellaropoulos GC, Nikiforidis GC, Siablis D. $\mathrm{CT}$ angiography with three-dimensional techniques for the early diagnosis of intracranial aneurysms. Comparison with intra-arterial DSA and the surgical findings. European journal of radiology. 2004;49(3):212-23.

11. Chen W, Wang J, Xing W, Xu Q, Qiu J, Huang Q, Sun Y, Yu S, Peng Y. Accuracy of 16-row multislice computerized tomography angiography for assessment of intracranial aneurysms. Surgical neurology. 2009;71(1):32-42

12. Taschner CA, Thines L, Lernout M, Lejeune JP, Leclerc X. Treatment decision in ruptured intracranial aneurysms: comparison between multi-detector row CT angiography and digital subtraction angiography. Journal of Neuroradiology. 2007;34(4):243-9.

13. Anderson GB, Steinke DE, Petruk KC, Ashforth R, Findlay JM. Computed tomographic angiography versus digital subtraction angiography for the diagnosis and early treatment of ruptured intracranial aneurysms. Neurosurgery. 1999; 45(6): 1315-22

14. Velthuis BK, van Leeuwen MS, Witkamp TD, Ramos LM, van der Sprenkel JW, Rinkel GJ. Computerized tomography angiography in patients with subarachnoid hemorrhage: from aneurysm detection to treatment without conventional angiography. Journal of neurosurgery. 1999;91(5):761-7.

15. Struffert T, Doelken M, Adamek E, Schwarz M, Engelhorn T, Kloska S, Ott S, Doerfler A. Flat-detector computed tomography with intravenous contrast material application in experimental aneurysms: comparison with multislice $\mathrm{CT}$ and conventional angiography. Acta Radiologica. 2010;51(4):431-7.

16. Donmez H, Serifov E, Kahriman G, Mavili E, Durak AC, 
Menkü A. Comparison of 16-row multislice CT angiography with conventional angiography for detection and evaluation of intracranial aneurysms. European journal of radiology. 2011; 80(2):455-61

17. Tipper G, U-King-Im JM, Price SJ, Trivedi RA, Cross JJ, Higgins NJ, Farmer R, Wat J, Kirollos R, Kirkpatrick PJ, Antoun NM. Detection and evaluation of intracranial aneurysms with 16-row multislice CT angiography. Clinical radiology. 2005;60(5):565-72.

18. Yoon DY, Hwang HS, Chang SK, Rho YS, Ahn HY, Kim JH, Lee IJ. CT, MR, US, 18 F-FDG PET/CT, and their combined use for the assessment of cervical lymph node metastases in squamous cell carcinoma of the head and neck. European radiology. 2009;19(3):634-42

19. White PM, Wardlaw JM, Easton V. Can noninvasive imaging accurately depict intracranial aneurysms? A systematic review. Radiology. 2000;217(2):361-70.

20. Westerlaan HE, Van Dijk JM, Jansen-van der Weide MC, de Groot JC, Groen RJ, Mooij JJ, Oudkerk M. Intracranial aneurysms in patients with subarachnoid hemorrhage: CT angiography as a primary examination tool for diagnosis - systematic review and meta-analysis. Radiology. 2011;258(1):134-45 